\title{
Boundedness of commutators of an oscillatory integral operator
}

by

Xia Xia (Tianjin) and Shanzhen Lu (Beijing)

\begin{abstract}
We obtain a necessary and sufficient condition for $L^{p}$ boundedness of commutators of certain oscillatory integral operators and Lipschitz functions.
\end{abstract}

1. Introduction and result. The following oscillatory integral operator is closely related to the Bochner-Riesz operator below the critical index (see [5]). Set $K(x)=e^{i|x|^{a}} /|x|^{\alpha}, x \in \mathbb{R}^{n} \backslash\{0\}$, where $a>0, a \neq 1$ and $0<\alpha<n$. Then $K$ belongs to the space $\mathcal{S}^{\prime}\left(\mathbb{R}^{n}\right)$ of tempered distributions and we set

$$
T f=K * f, \quad f \in \mathrm{C}_{0}^{\infty}\left(\mathbb{R}^{n}\right) .
$$

In the case $n=1$, Sampson, Naparstek and Drobot [4] obtained some $L^{p}$ boundedness properties of $T$. In higher dimensions, there is a well known result due to Sjölin [5].

Theorem A ([5]). If $\alpha \geq n(1-a / 2)$ and $p_{0}=n a /(n a-n+\alpha)$, then $T$ is bounded on $L^{p}\left(\mathbb{R}^{n}\right)$ if and only if $p_{0} \leq p \leq p_{0}^{\prime}$. If $\alpha<n(1-a / 2)$, then $T$ is not bounded on any $L^{p}\left(\mathbb{R}^{n}\right), 1 \leq p \leq \infty$.

For $\beta>0$, the homogeneous Lipschitz space $\dot{\Lambda}_{\beta}$ is the space of functions $f$ such that

$$
\|f\|_{\dot{\Lambda}_{\beta}}=\sup _{x, h \in \mathbb{R}^{n}, h \neq 0} \frac{\left|\Delta_{h}^{[\beta]+1} f(x)\right|}{|h|^{\beta}}<\infty,
$$

where $\Delta_{h}^{k}$ denotes the $k$ th difference operator. It is obvious that when $0<$ $\beta<1, f \in \dot{\Lambda}_{\beta}$ implies $|f(x)-f(y)| \leq|x-y|^{\beta}\|f\|_{\dot{\Lambda}_{\beta}}$ for all $x, y \in \mathbb{R}^{n}$. We focus on the case $0<\beta<1$.

2000 Mathematics Subject Classification: Primary 42B20; Secondary $42 B 35$.

Key words and phrases: commutator, oscillatory integral operator, Lipschitz function.

This project is supported by NNFS of China (10571014). The second author is the corresponding author. 
Let $T$ be a linear operator. Then the commutator of $T$ and a Lipschitz function $b$ is defined by

$$
[b, T] f(x)=b(x) T f(x)-T(b f)(x) .
$$

If $T$ is a singular integral operator, Janson [2] showed that the condition $b \in \dot{\Lambda}_{\beta}$ is equivalent to $\left(L^{p}, L^{q}\right)$ boundedness of $[b, T]$. Later, Paluszyński [3] proved that if $T$ is the Riesz potential operator, then the condition $b \in$ $\dot{\Lambda}_{\beta}$ characterizes the boundedness of $[b, T]$ from $L^{p}$ to a certain TriebelLizorkin space. However, their method of proof is only suitable for operators $T$ bounded on $L^{p}$ for all $1<p<\infty$, but not for those bounded on $L^{p}$ only for some $p$. In this paper, we will study the $L^{p}$ boundedness of commutators of the oscillatory integral operator mentioned above and Lipschitz functions using a method called scale changing. This method was first introduced by Carleson and Sjölin [1], who proved that the Bochner-Riesz operator below the critical index is bounded on some $L^{p}\left(\mathbb{R}^{2}\right)$. To do this, they considered a class of oscillatory integrals.

Let $\Psi: \mathbb{R}^{n} \times \mathbb{R}^{n} \rightarrow \mathbb{C}$ be a smooth function of compact support in $x$ and $y$, and let $\Phi: \mathbb{R}^{n} \times \mathbb{R}^{n} \rightarrow \mathbb{R}$ be smooth. Assume that on the support of $\Psi$, the Hessian determinant of $\Phi$ is nonvanishing, i.e.

$$
\operatorname{det}\left(\frac{\partial^{2} \Phi(x, y)}{\partial x_{i} \partial y_{j}}\right) \neq 0 \text {. }
$$

We consider the oscillatory integral

$$
\left(T_{\lambda} f\right)(y)=\int_{\mathbb{R}^{n}} e^{i \lambda \Phi(x, y)} \Psi(x, y) f(x) d x .
$$

Then we have

TheOrem B ([6]). Under the above assumptions on $\Phi$ and $\Psi$,

$$
\left\|T_{\lambda} f\right\|_{L^{2}\left(\mathbb{R}^{n}\right)} \leq C \lambda^{-n / 2}\|f\|_{L^{2}\left(\mathbb{R}^{n}\right)} .
$$

Obviously, we also have

$$
\left\|T_{\lambda} f\right\|_{L^{\infty}\left(\mathbb{R}^{n}\right)} \leq C\|f\|_{L^{\infty}\left(\mathbb{R}^{n}\right)}, \quad\left\|T_{\lambda} f\right\|_{L^{1}\left(\mathbb{R}^{n}\right)} \leq C\|f\|_{L^{1}\left(\mathbb{R}^{n}\right)} .
$$

By interpolation,

$$
\begin{array}{ll}
\left\|T_{\lambda} f\right\|_{L^{p}\left(\mathbb{R}^{n}\right)} \leq C \lambda^{-n / p}\|f\|_{L^{p}\left(\mathbb{R}^{n}\right)}, & 2 \leq p<\infty, \\
\left\|T_{\lambda} f\right\|_{L^{p}\left(\mathbb{R}^{n}\right)} \leq C \lambda^{-n / p^{\prime}}\|f\|_{L^{p}\left(\mathbb{R}^{n}\right)}, & 1 \leq p<2,1 / p+1 / p^{\prime}=1 .
\end{array}
$$

In [5], the author proved the sufficiency part of Theorem A by using multiplier theorems on $H^{p}$ spaces. But this method cannot be applied to our commutators. This is not surprising since the Fourier transform is not well defined for Lipschitz functions. In this paper, we use the scale changing method to obtain a sufficient condition for $L^{p}$ boundedness of the commutator mentioned above. To get a necessary condition for $L^{p}$ boundedness of 
those commutators, we follow the method in [5] together with choosing a proper Lipschitz function. Our main result can be stated as follows.

Theorem. Let $a>0, a \neq 1,0<\beta<1, n(1-a / 2)+\beta \leq \alpha<n$, and set $p_{0}=n a /(n a-n+\alpha-\beta)$. Then the commutator $[b, T]$ defined by (1.1) is bounded on $L^{p}\left(\mathbb{R}^{n}\right)$, for all $b \in \dot{\Lambda}_{\beta}\left(\mathbb{R}^{n}\right)$, with the operator norm $\leq C\|b\|_{\dot{\Lambda}_{\beta}\left(\mathbb{R}^{n}\right)}$, if and only if $p_{0} \leq p \leq p_{0}^{\prime}$. If $\alpha<n(1-a / 2)+\beta$, then there exists $a b \in \dot{\Lambda}_{\beta}\left(\mathbb{R}^{n}\right)$ such that $[b, T]$ is not bounded on any $L^{p}\left(\mathbb{R}^{n}\right)$, $1 \leq p \leq \infty$.

2. Proof of Theorem. First of all, let us prove that $p_{0} \leq p \leq p_{0}^{\prime}$ is a necessary condition for the boundedness of $[b, T]$ on $L^{p}$ for any $b \in \dot{\Lambda}_{\beta}$. We only give the proof of $p_{0} \leq p$. We first treat the case $a>1,0<$ $\beta<1, n(1-a / 2)+\beta \leq \alpha$. If we set $b_{0}(x)=|x|^{\beta}$, then $b_{0} \in \dot{\Lambda}_{\beta}\left(\mathbb{R}^{n}\right)$ and $\left\|b_{0}\right\|_{\dot{\Lambda}_{\beta}}=1$. Since for any $b \in \dot{\Lambda}_{\beta},[b, T]$ is bounded on $L^{p}$, the operator $\left[b_{0}, T\right]$ is then bounded on $L^{p}$. Now we assume that $\left[b_{0}, T\right]$ is bounded on $L^{p}\left(\mathbb{R}^{n}\right)$, where $1 \leq p \leq 2$. We shall prove that $p \geq p_{0}=n a /(n a-n+\alpha-\beta)>1$. Choose $\psi \in \mathrm{C}_{0}^{\infty}\left(\mathbb{R}^{n}\right)$ such that $0 \leq \psi \leq 1, \psi(x)=1$ for $|x| \leq 1 / 2$ and $\psi(x)=0$ for $|x| \geq 1$. Let $\varepsilon>0$ be small enough and $\psi_{\varepsilon}(x)=\psi(x / \varepsilon)$. Then

$$
\left\|\psi_{\varepsilon}\right\|_{p}^{p}=\int_{\mathbb{R}^{n}}\left|\psi_{\varepsilon}(x)\right|^{p} d x=C \varepsilon^{n}
$$

for some constant $C>0$. When $\varepsilon$ is small enough,

$$
\begin{aligned}
M & =\left\{k \in \mathbb{N}: \frac{1}{2 \pi}\left(\frac{96 a}{\pi} \cdot \varepsilon\right)^{-a /(a-1)}+\frac{1}{6} \leq k \leq \frac{1}{2 \pi}\left(\frac{12 a}{\pi} \cdot \varepsilon\right)^{-a /(a-1)}-\frac{1}{6}\right\} \\
& \neq \emptyset .
\end{aligned}
$$

For $k \in M$, let

$$
\begin{aligned}
I_{k} & =\left[(2 k \pi-\pi / 3)^{1 / a}+\varepsilon,(2 k \pi+\pi / 3)^{1 / a}-\varepsilon\right], \\
A_{k} & =\left[(2 k \pi+\pi / 3)^{1 / a}-\varepsilon,(2(k+1) \pi-\pi / 3)^{1 / a}+\varepsilon\right] .
\end{aligned}
$$

Clearly, $I_{k} \cap I_{k+1}=\emptyset$. Furthermore, using the differential intermediate value theorem, for some $0<\theta, \vartheta<1$ and all $k \in M$, we get

$$
\begin{aligned}
\left|A_{k}\right| & =2 \varepsilon+(2(k+1) \pi-\pi / 3)^{1 / a}-(2 k \pi+\pi / 3)^{1 / a} \\
& =2 \varepsilon+\frac{1}{a} \cdot \frac{4 \pi}{3}[\theta(2(k+1) \pi-\pi / 3)+(1-\theta)(2 k \pi+\pi / 3)]^{1 / a-1} \\
& \leq 2 \varepsilon+\frac{4 \pi}{3 a}(2 k \pi+\pi / 3)^{1 / a-1} \leq 3\left[\frac{2 \pi}{3 a}(2 k \pi+\pi / 3)^{1 / a-1}-2 \varepsilon\right] \\
& \leq 3\left\{\frac{1}{a} \cdot \frac{2 \pi}{3}[\vartheta(2 k \pi-\pi / 3)+(1-\vartheta)(2 k \pi+\pi / 3)]^{1 / a-1}-2 \varepsilon\right\} \\
& \leq 3\left[(2 k \pi+\pi / 3)^{1 / a}-(2 k \pi-\pi / 3)^{1 / a}-2 \varepsilon\right]=3\left|I_{k}\right| .
\end{aligned}
$$


It follows that

$$
\begin{aligned}
& \int_{|x| \in A_{k}}|x|^{-\alpha p} d x \leq C\left((2 k \pi+\pi / 3)^{1 / a}-\varepsilon\right)^{-\alpha p} \int_{A_{k}} r^{n-1} d r \\
& \leq C\left((2 k \pi+\pi / 3)^{1 / a}-\varepsilon\right)^{-\alpha p}\left[(2(k+1) \pi-\pi / 3)^{1 / a}+\varepsilon\right]^{n-1}\left|A_{k}\right| \\
& \leq C\left((2 k \pi+\pi / 3)^{1 / a}-\varepsilon\right)^{-\alpha p}\left[(3(2 k \pi-\pi / 3))^{1 / a}+\varepsilon\right]^{n-1} \cdot 3\left|I_{k}\right| \\
& \leq C 3^{1+(n-1) / a} \int_{I_{k}} r^{-\alpha p+n-1} d r=C \int_{|x| \in I_{k}}|x|^{-\alpha p} d x,
\end{aligned}
$$

where $C$ is a constant independent of $k$. Moreover, if we set

$$
\begin{aligned}
& k_{0}=\min \{k: k \in M\} \leq \frac{1}{2 \pi}\left(\frac{96 a}{\pi} \cdot \varepsilon\right)^{-a /(a-1)}+\frac{1}{6}+1, \\
& k_{1}=\max \{k: k \in M\} \geq \frac{1}{2 \pi}\left(\frac{12 a}{\pi} \cdot \varepsilon\right)^{-a /(a-1)}+\frac{1}{6}-1,
\end{aligned}
$$

then

$$
\begin{aligned}
& \left(2 k_{0} \pi-\pi / 3\right)^{1 / a}+\varepsilon \leq\left[\left(\frac{96 a}{\pi} \cdot \varepsilon\right)^{-a /(a-1)}+2 \pi\right]^{1 / a}+\varepsilon \leq\left(\frac{48 a}{\pi} \cdot \varepsilon\right)^{-1 /(a-1)}, \\
& \left(2 k_{1} \pi+\pi / 3\right)^{1 / a}-\varepsilon \geq\left[\left(\frac{12 a}{\pi} \cdot \varepsilon\right)^{-a /(a-1)}-2 \pi\right]^{1 / a}-\varepsilon \geq\left(\frac{24 a}{\pi} \cdot \varepsilon\right)^{-1 /(a-1)} .
\end{aligned}
$$

It follows that

$$
\left[\left(\frac{48 a}{\pi} \cdot \varepsilon\right)^{-1 /(a-1)},\left(\frac{24 a}{\pi} \cdot \varepsilon\right)^{-1 /(a-1)}\right] \subset \bigcup_{k \in M}\left(I_{k} \cup A_{k}\right) .
$$

Thus, setting $C_{1}=24 a / \pi$, by (2.1) and (2.2),

$$
\begin{gathered}
\int_{\left(2 C_{1} \varepsilon\right)^{-1 /(a-1)} \leq|x| \leq\left(C_{1} \varepsilon\right)^{-1 /(a-1)}}|x|^{-\alpha p} d x \\
\leq \sum_{k \in M} \int_{|x| \in I_{k}}|x|^{-\alpha p} d x+\sum_{k \in M} \int_{|x| \in A_{k}}|x|^{-\alpha p} d x \leq C \sum_{k \in M} \int_{|x| \in I_{k}}|x|^{-\alpha p} d x .
\end{gathered}
$$

For each $|x| \in I_{k}$, we have $2 k \pi-\pi / 3 \leq(|x|-\varepsilon)^{a}$ and $(|x|+\varepsilon)^{a} \leq 2 k \pi+\pi / 3$. It follows that $(|x|-\varepsilon)^{a} \leq|y|^{a} \leq(|x|+\varepsilon)^{a}$ for all $y \in\{y:|x-y| \leq \varepsilon\}$, which implies

$$
1 / 2 \leq \cos \left(|y|^{a}\right) \leq 1, \quad|y|^{\alpha} \leq(2|x|)^{\alpha}
$$

Hence

$$
\operatorname{Re}\left(K * \psi_{\varepsilon}\right)(x)=\int_{\mathbb{R}^{n}} \frac{\cos \left(|y|^{a}\right)}{|y|^{\alpha}} \psi_{\varepsilon}(x-y) d y \geq \frac{C}{|x|^{\alpha}} \int_{|x-y| \leq \varepsilon} \psi_{\varepsilon}(x-y) d y=\frac{C \varepsilon^{n}}{|x|^{\alpha}}
$$


Since $|x| \geq\left(4 C_{1} \varepsilon\right)^{-/(a-1)}$ for $|x| \in I_{k}, k \in M$, we have

$$
\begin{aligned}
\left|\left[b_{0}, T\right] \psi_{\varepsilon}(x)\right| & =\left|\int_{\mathbb{R}^{n}} \frac{e^{i|y|^{a}}}{|y|^{\alpha}}\left[b_{0}(x)-b_{0}(x-y)\right] \psi_{\varepsilon}(x-y) d y\right| \\
& \geq \int_{|x-y| \leq \varepsilon} \frac{\cos \left(|y|^{a}\right)}{|y|^{\alpha}}\left[|x|^{\beta}-|x-y|^{\beta}\right] \psi_{\varepsilon}(x-y) d y \\
& \geq C\left(\left(4 C_{1} \varepsilon\right)^{-\beta /(a-1)}-\varepsilon^{\beta}\right) \operatorname{Re}\left(K * \psi_{\varepsilon}\right)(x) \\
& \geq C\left(8 C_{1} \varepsilon\right)^{-\beta /(a-1)} \operatorname{Re}\left(K * \psi_{\varepsilon}\right)(x) \geq C \frac{\varepsilon^{-\beta /(a-1)+n}}{|x|^{\alpha}} .
\end{aligned}
$$

Therefore, using (2.3) and (2.4), we get

$$
\begin{aligned}
\left\|\left[b_{0}, T\right] \psi_{\varepsilon}\right\|_{p}^{p} & =\int_{\mathbb{R}^{n}}\left|\left[b_{0}, T\right] \psi_{\varepsilon}(x)\right|^{p} d x \geq \sum_{k \in M} \int_{|x| \in I_{k}}\left|\left[b_{0}, T\right] \psi_{\varepsilon}(x)\right|^{p} d x \\
& \geq C \sum_{k \in M} \int_{|x| \in I_{k}} \frac{\varepsilon^{[-\beta /(a-1)+n] p}}{|x|^{\alpha p}} d x \\
& \geq C \varepsilon^{[-\beta(a-1)+n] p}|x|^{-\alpha p} d x \\
& \geq C \varepsilon^{[-\beta /(a-1)+n] p} \varepsilon^{-(n-\alpha p) /(a-1)} .
\end{aligned}
$$

Since $\left[b_{0}, T\right]$ is bounded on $L^{p}$, it follows that

$$
\varepsilon^{[-\beta /(a-1)+n] p-(n-\alpha p) /(a-1)} \leq C \varepsilon^{n},
$$

and this can hold for small values of $\varepsilon$ only if

$$
[-\beta /(a-1)+n] p-(n-\alpha p) /(a-1) \geq n .
$$

That is,

$$
p(n a-n+\alpha-\beta) \geq n a .
$$

The inequality $\alpha \geq n(1-a / 2)+\beta$ yields $n a-n+\alpha-\beta \geq n a / 2>0$, and we conclude that

$$
p \geq p_{0}=\frac{n a}{n a-n+\alpha-\beta} .
$$

Thus $p_{0} \leq p \leq p_{0}^{\prime}$ is a necessary condition for the boundedness of $[b, T]$ on $L^{p}$.

We next study the case $a>1,0<\beta<1, \alpha<n(1-a / 2)+\beta$. As above, let $b_{0}(x)=|x|^{\beta}$. If $\left[b_{0}, T\right]$ is bounded on $L^{p}$ with $1 \leq p \leq 2$, then we obtain the inequality (2.5) as above. Using the condition on $\alpha$ we conclude that

$$
p \geq \frac{n a}{n a-n+\alpha-\beta}>2,
$$

which gives a contradiction. Similarly, we can prove that $\left[b_{0}, T\right]$ is not bounded on $L^{p}$ with $2<p<\infty$. Hence $\left[b_{0}, T\right]$ is not bounded on any $L^{p}$ in this case. 
We shall use an argument similar to the above to prove the necessity in the case $0<a<1,0<\beta<1, n(1-a / 2)+\beta \leq \alpha<n$. We shall prove that $p_{0} \leq p \leq p_{0}^{\prime}$ where $p_{0}=n a /(n a-n+\alpha-\beta)>1$. Let $\psi_{\lambda}(x)=\psi(x / \lambda), \lambda>0$. Take $\lambda$ large enough. There exists $k_{0} \in \mathbb{N}$ such that

$$
\begin{aligned}
& k_{0}>\frac{1}{2 \pi}\left(\frac{12 a \lambda}{\pi}\right)^{a /(1-a)}+\frac{1}{6}, \\
& k_{0}>\frac{1}{2 \pi}\left(\lambda^{\beta /(1-a)}+\lambda^{\beta}\right)^{a / \beta}+\frac{1}{6}, \\
& k_{0}<\frac{1}{2 \pi}\left[(4 \lambda)^{1 /(1-a)}-\lambda\right]^{a}+\frac{1}{6} .
\end{aligned}
$$

Then, for all $k \geq k_{0}$, set

$$
\begin{aligned}
I_{k} & =\left[(2 k \pi-\pi / 3)^{1 / a}+\lambda,(2 k \pi+\pi / 3)^{1 / a}-\lambda\right] \\
A_{k} & =\left[(2(k-1) \pi+\pi / 3)^{1 / a}-\lambda,(2 k \pi-\pi / 3)^{1 / a}+\lambda\right] .
\end{aligned}
$$

Clearly $I_{k} \cap I_{k+1}=\emptyset$. Furthermore, $I_{k} \neq \emptyset$, because using the differential intermediate value theorem and (2.6), we have

$$
\begin{aligned}
\left|I_{k}\right| & =(2 k \pi+\pi / 3)^{1 / a}-(2 k \pi-\pi / 3)^{1 / a}-2 \lambda \\
& \geq \frac{1}{a} \cdot \frac{2 \pi}{3}(2 k \pi-\pi / 3)^{1 / a-1}-2 \lambda>0 .
\end{aligned}
$$

Since by (2.6),

$$
\begin{aligned}
\left|A_{k}\right| & =(2 k \pi-\pi / 3)^{1 / a}-(2(k-1) \pi+\pi / 3)^{1 / a}+2 \lambda \\
& \leq \frac{1}{a} \cdot \frac{4 \pi}{3}(2 k \pi-\pi / 3)^{1 / a-1}+2 \lambda \\
& \leq 3\left[\frac{1}{a} \cdot \frac{2 \pi}{3}(2 k \pi-\pi / 3)^{1 / a-1}-2 \lambda\right] \leq 3\left|I_{k}\right|,
\end{aligned}
$$

we have

$$
\begin{aligned}
& \int_{|x| \in A_{k}}|x|^{-\alpha p} d x \\
\leq & C\left[(2(k-1) \pi+\pi / 3)^{1 / a}-\lambda\right]^{-\alpha p} \int_{A_{k}} r^{n-1} d r \\
\leq & C 2^{\alpha p / a}\left[(2(2 k \pi-5 \pi / 3))^{1 / a}-2^{1 / a} \lambda\right]^{-\alpha p}\left[(2 k \pi-\pi / 3)^{1 / a}+\lambda\right]^{n-1}\left|A_{k}\right| \\
\leq & C\left[(2 k \pi+\pi / 3)^{1 / a}-2^{1 / a} \lambda\right]^{-\alpha p}\left[(2 k \pi-\pi / 3)^{1 / a}+\lambda\right]^{n-1} \cdot 3\left|I_{k}\right| \\
\leq & C 2^{\alpha p}\left[(2 k \pi+\pi / 3)^{1 / a}-\lambda\right]^{-\alpha p} \int_{I_{k}} r^{n-1} d r \\
\leq & C \int_{I_{k}} r^{-\alpha p+n-1} d r=C \int_{|x| \in I_{k}}|x|^{-\alpha p} d x,
\end{aligned}
$$


where $C$ is independent of $k$. Moreover, by (2.8),

$$
\left[(4 \lambda)^{1 /(1-a)},+\infty\right) \subset \bigcup_{k \geq k_{0}}\left(I_{k} \cup A_{k}\right) .
$$

It follows from (2.9) that

$$
\begin{aligned}
\int_{|x| \geq(4 \lambda)^{1 /(1-a)}}|x|^{-\alpha p} & \leq \sum_{k \geq k_{0}} \int_{|x| \in I_{k}}|x|^{-\alpha p} d x+\sum_{k \geq k_{0}} \int_{|x| \in A_{k}}|x|^{-\alpha p} d x \\
& \leq C \sum_{k \geq k_{0}} \int_{|x| \in I_{k}}|x|^{-\alpha p} d x .
\end{aligned}
$$

For each $|x| \in I_{k}$, we have $2 k \pi-\pi / 3 \leq(|x|-\lambda)^{a}$ and $(|x|+\lambda)^{a} \leq 2 k \pi+\pi / 3$. It follows that for all $|x-y| \leq \lambda$,

$$
1 / 2 \leq \cos \left(|y|^{a}\right) \leq 1, \quad|y|^{\alpha} \leq C|x|^{\alpha} .
$$

Hence

$$
\begin{aligned}
\operatorname{Re}\left(K * \psi_{\lambda}\right)(x) & =\int_{\mathbb{R}^{n}} \frac{\cos \left(|y|^{a}\right)}{|y|^{\alpha}} \psi_{\lambda}(x-y) d y \\
& \geq \frac{C}{|x|^{\alpha}} \int_{|x-y| \leq \lambda} \psi_{\lambda}(x-y) d y=\frac{C \lambda^{n}}{|x|^{\alpha}} .
\end{aligned}
$$

Moreover, it follows from (2.7) that for $|x| \in I_{k}, k \geq k_{0}$, we have

$$
|x|^{\beta}-\lambda^{\beta} \geq\left[\left(2 k_{0} \pi-\pi / 3\right)^{1 / a}+\lambda\right]^{\beta}-\lambda^{\beta} \geq \lambda^{\beta /(1-a)} .
$$

Therefore,

$$
\begin{aligned}
\left|\left[b_{0}, T\right] \psi_{\lambda}(x)\right| & \geq \int_{|x-y| \leq \lambda} \frac{\cos \left(|y|^{a}\right)}{|y|^{\alpha}}\left[|x|^{\beta}-|x-y|^{\beta}\right] \psi_{\lambda}(x-y) d y \\
& \geq C \lambda^{\beta /(1-a)} \operatorname{Re}\left(K * \psi_{\lambda}\right)(x)=C \frac{\lambda^{n+\beta /(1-a)}}{|x|^{\alpha}} .
\end{aligned}
$$

Consequently, together with (2.10),

$$
\begin{aligned}
\left\|\left[b_{0}, T\right] \psi_{\lambda}\right\|_{p}^{p} & =\int_{\mathbb{R}^{n}}\left|\left[b_{0}, T\right] \psi_{\lambda}(x)\right|^{p} d x \geq \sum_{k \geq k_{0}} \int_{|x| \in I_{k}}\left|\left[b_{0}, T\right] \psi_{\lambda}(x)\right|^{p} d x \\
& \geq C \sum_{k \geq k_{0}} \int_{|x| \in I_{k}} \frac{\lambda^{[n+\beta /(1-a)] p}}{|x|^{\alpha p}} d x \\
& \geq C \lambda^{[n+\beta /(1-a)] p} \int_{|x| \geq(4 \lambda)^{1 /(1-a)}}|x|^{-\alpha p} d x \\
& \geq C \lambda^{[n+\beta /(1-a)] p} \lambda^{(n-\alpha p) /(1-a)}
\end{aligned}
$$


where $n<\alpha p$. Since $\left[b_{0}, T\right]$ is bounded on $L^{p}$, it follows that

$$
\lambda^{[n+\beta /(1-a)] p+(n-\alpha p) /(1-a)} \leq C \lambda^{n} .
$$

This can hold for large values of $\lambda$ only if $[n+\beta /(1-a)] p+(n-\alpha p) /(1-a)$ $\leq n$. That is,

$$
p(n a-n+\alpha-\beta) \geq n a .
$$

The inequality $\alpha \geq n(1-a / 2)+\beta$ yields $n a-n+\alpha-\beta \geq n a / 2>0$, and we conclude that

$$
p \geq p_{0}=\frac{n a}{n a-n+\alpha-\beta} .
$$

Thus $p_{0} \leq p \leq p_{0}^{\prime}$ is a necessary condition for the boundedness of $[b, T]$ on $L^{p}$.

In the case $0<a<1,0<\beta<1, \alpha<n(1-a / 2)+\beta$, if $\left[b_{0}, T\right]$ is bounded on $L^{p}$ with $1 \leq p \leq 2$, then we obtain the inequality (2.11) as above. Invoking the condition on $\alpha$ we obtain

$$
p \geq \frac{n a}{n a-n+\alpha-\beta}>2,
$$

which gives a contradiction. Similarly, we can prove that $\left[b_{0}, T\right]$ is not bounded on $L^{p}$ with $p>2$ in this case.

It remains to prove that $p_{0} \leq p \leq p_{0}^{\prime}$ is a sufficient condition for $L^{p}$ boundedness of $[b, T]$, where $b \in \dot{\Lambda}_{\beta}$. We treat $p_{0}<p<p_{0}^{\prime}$ first. The proof of $L^{p}$ boundedness for $[b, T]$ can be reduced to showing that for $\lambda$ large enough, we have

$$
\int_{[0, \lambda]^{n}}\left|\int_{[0, \lambda]^{n}}[b(x)-b(y)] \frac{e^{i|x-y|^{a}}}{|x-y|^{\alpha}} f(y) d y\right|^{p} d x \leq C\|b\|_{\dot{\Lambda}_{\beta}}^{p} \int_{[0, \lambda]^{n}}|f(x)|^{p} d x,
$$

where $C$ is a constant independent of $\lambda$ and $f$. Let $I=[0,1]^{n}$ be the unit cube in $\mathbb{R}^{n}$. By changing variable, our goal is to prove

$$
\int_{I}\left|\lambda^{n-\alpha} \int_{I}[b(\lambda x)-b(\lambda y)] \frac{e^{i \lambda^{a}|x-y|^{a}}}{|x-y|^{\alpha}} f(\lambda y) d y\right|^{p} d x \leq C\|b\|_{\dot{\Lambda}_{\beta}}^{p} \int_{I}|f(\lambda x)|^{p} d x .
$$

Set

$$
S_{\lambda}^{b} f(x)=\lambda^{n-\alpha+\beta} \int_{I}[b(x)-b(y)] \frac{e^{i \lambda^{a}|x-y|^{a}}}{|x-y|^{\alpha}} f(y) d y .
$$

Noting that $\|b(\lambda \cdot)\|_{\dot{\Lambda}_{\beta}}=\lambda^{\beta}\|b\|_{\dot{\Lambda}_{\beta}}$, it suffices to show

$$
\begin{gathered}
\left\|S_{\lambda}^{b} f\right\|_{L^{p}(I)} \leq C\|b\|_{\dot{\Lambda}_{\beta}}\|f\|_{L^{p}(I)}, \\
\frac{n a}{n a-n+\alpha-\beta}<p<\frac{n a}{n-\alpha+\beta} .
\end{gathered}
$$

Let $\Omega_{k}, k=0,1, \ldots$, denote the set of all dyadic cubes in $[-2,2]^{n}$ with side length $2^{-k}$, and $\Omega_{k}^{*}$ the set of all cubes which are unions of $2^{n}$ cubes 
in $\Omega_{k}$. For each $\omega^{*} \in \Omega_{k}^{*}$, its side length is $2^{-k+1}$. If $x \in I$ and $x$ does not belong to the boundary of any dyadic cube, then there exists a unique cube $\omega_{k}^{*}(x) \in \Omega_{k}^{*}$ such that $x \in \frac{1}{2} \omega_{k}^{*}(x)$. Let $\omega_{-1}^{*}=[-2,2]^{n}$. For a measurable set $D$, write

$$
\begin{aligned}
E(x, D) & =\lambda^{n-\alpha+\beta} \int_{D}[b(x)-b(y)] \frac{e^{i \lambda^{a}|x-y|^{a}}}{|x-y|^{\alpha}} f(y) d y, \quad x \in I, \\
E_{k}(x) & =E\left(x,\left[\omega_{k-1}^{*}(x) \backslash \omega_{k}^{*}(x)\right] \cap I\right), \quad k \geq 0 .
\end{aligned}
$$

Then we get

$$
S_{\lambda}^{b} f(x)=\sum_{k=0}^{\infty} E_{k}(x)=\sum_{k=0}^{k_{N}} E_{k}(x)+\sum_{k=k_{N}+1}^{\infty} E_{k}(x),
$$

where $2^{-k_{N}}<\lambda^{-1} \leq 2^{-k_{N}+1}$. Note that for $k$ large enough we must have $\omega_{k}^{*}(x) \subset I$, so we may assume $E_{k}(x)=E\left(x, \omega_{k-1}^{*} \backslash \omega_{k}^{*}(x)\right)$. It follows from the construction that $\omega_{k-1}^{*}(x) \backslash \omega_{k}^{*}(x)$ is made up of $4^{n}-2^{n}$ cubes with side length $2^{-k}$. If we set $F(\omega)=4 \omega \backslash 2 \omega$, then $\sum_{\omega \in \Omega_{k}} \chi_{F(\omega)}(x) \leq 4^{n}-2^{n}$. Hölder's inequality yields

$$
\left|E_{k}(x)\right|^{p} \leq C \sum_{\omega \in \Omega_{k}}|E(x, \omega)|^{p} \chi_{F(\omega)}(x) .
$$

Therefore, for any $k$,

$$
\int_{I}\left|E_{k}(x)\right|^{p} d x \leq C \sum_{\omega \in \Omega_{k}} \int_{F(\omega)}|E(x, \omega)|^{p} d x
$$

where the constant $C$ only depends on $n, p$. Let $x_{\omega}$ be the point of $\omega \in \Omega_{k}$ such that $x_{i} \leq y_{i}, i=1, \ldots, n$, for all $y=\left(y_{1}, \ldots, y_{n}\right) \in \omega$.

When $k \geq k_{N}+1$,

$$
\begin{aligned}
& \int_{F(\omega)}|E(x, \omega)|^{p} d x \\
& \quad=2^{-n k} \int_{F(I)}\left(\lambda^{n-\alpha+\beta} 2^{-k(n-\alpha)} \int_{I}\left|b\left(2^{-k} x+x_{\omega}\right)-b\left(2^{-k} y+x_{\omega}\right)\right|\right. \\
& \quad \leq C\left(2^{-k} \lambda\right)^{p(n-\alpha+\beta)}\|b\|_{\dot{\Lambda}_{\beta}}^{p}\|f\|_{L^{p}(\omega)}^{p} \\
& \quad \leq C\left(2^{-k+k_{N}}\right)^{p(n-\alpha+\beta)}\|b\|_{\dot{\Lambda}_{\beta}}^{p}\|f\|_{L^{p}(\omega)}^{p} .
\end{aligned}
$$


Thus

$$
\begin{aligned}
\sum_{k=k_{N}+1}^{\infty}\left\|E_{k}\right\|_{L^{p}(I)} & \leq C \sum_{k=k_{N}+1}^{\infty}\left(2^{-k+k_{N}}\right)^{n-\alpha+\beta}\|b\|_{\dot{\Lambda}_{\beta}}\|f\|_{L^{p}(I)} \\
& \leq C\|b\|_{\dot{\Lambda}_{\beta}}\|f\|_{L^{p}(I)} .
\end{aligned}
$$

We consider the case $0 \leq k \leq k_{N}$. Recalling that the side length of $\omega$ is $2^{-k}$, we have

$$
\begin{aligned}
& \int|E(x, \omega)|^{p} d x \\
& F(\omega) \\
& =\int_{F(\omega)}\left|\lambda^{n-\alpha+\beta} \int_{\omega}[b(x)-b(y)] \frac{e^{i \lambda^{a}|x-y|^{a}}}{|x-y|^{\alpha}} f(y) d y\right|^{p} d x \\
& \leq C \lambda^{p(n-\alpha+\beta)} \int_{F(\omega)}\left|b(x)-b\left(x_{\omega}\right)\right|^{p}\left|\int_{\omega} \frac{e^{i \lambda^{a}|x-y|^{a}}}{|x-y|^{\alpha}} f(y) d y\right|^{p} d x \\
& +C \lambda^{p(n-\alpha+\beta)} \int_{F(\omega)}\left|\int_{\omega} \frac{e^{i \lambda^{a}|x-y|^{a}}}{|x-y|^{\alpha}}\left[b\left(x_{\omega}\right)-b(y)\right] f(y) d y\right|^{p} d x \\
& \leq C \lambda^{p(n-\alpha+\beta)} 2^{-k p \beta}\|b\|_{\dot{\Lambda}_{\beta}}^{p} \int_{F(\omega)-x_{\omega}}\left|\int_{\left[0,2^{-k}\right]^{n}} \frac{e^{i \lambda^{a}|x-y|^{a}}}{|x-y|^{\alpha}} f\left(y+x_{\omega}\right) d y\right|^{p} d x \\
& +C \lambda^{p(n-\alpha+\beta)} \int_{F(\omega)-x_{\omega}}\left|\int_{\left[0,2^{-k}\right]^{n}} \frac{e^{i \lambda^{a}|x-y|^{a}}}{|x-y|^{\alpha}}\left[b\left(x_{\omega}\right)-b\left(y+x_{\omega}\right)\right] f\left(y+x_{\omega}\right) d y\right|^{p} d x \\
& =C \lambda^{p(n-\alpha+\beta)} 2^{-k p(n-\alpha+\beta)} 2^{-k n}\|b\|_{\dot{\Lambda}_{\beta}}^{p} \int_{F(I)}\left|\int_{I} \frac{e^{i\left(2^{-k} \lambda\right)^{a}|x-y|^{a}}}{|x-y|^{\alpha}} f\left(2^{-k} y+x_{\omega}\right) d y\right|^{p} d x \\
& +C \lambda^{p(n-\alpha+\beta)} 2^{-k p(n-\alpha)-k n} \\
& \times \int_{F(I)}\left|\int_{I} \frac{e^{i\left(2^{-k} \lambda\right)^{a}|x-y|^{a}}}{|x-y|^{\alpha}}\left[b\left(x_{\omega}\right)-b\left(2^{-k} y+x_{\omega}\right)\right] f\left(2^{-k} y+x_{\omega}\right) d y\right|^{p} d x .
\end{aligned}
$$

In order to apply Theorem B, we set $\Phi(x, y)=|x-y|^{a}$ and let $\Psi$ be a smooth function defined on $F(I) \times I$. Without loss of generality, we consider $\Psi(x, y)=1 /|x-y|^{\alpha}$. Since

$$
\operatorname{det}\left(\frac{\partial^{2} \Phi(x, y)}{\partial x_{i} \partial y_{j}}\right)=(-a)^{n}(a-1)|x-y|^{n(a-2)},
$$

for $a \neq 1, x \in F(I)$ and $y \in I$ we have $\operatorname{det}\left(\frac{\partial^{2} \Phi(x, y)}{\partial x_{i} \partial y_{j}}\right) \neq 0$, as required in 
(1.2). When $2 \leq p$, using (1.3), we get

$$
\begin{aligned}
& \int_{F(\omega)} \mid E(x, \omega)^{p} d x \\
& \leq C \lambda^{p(n-\alpha+\beta)} 2^{-k p(n-\alpha+\beta)} 2^{-k n}\|b\|_{\dot{\Lambda}_{\beta}}^{p}\left(2^{-k} \lambda\right)^{-n a} \int_{I}\left|f\left(2^{-k} x+x_{\omega}\right)\right|^{p} d x \\
& \quad+C \lambda^{p(n-\alpha+\beta)} 2^{-k p(n-\alpha)} 2^{-k n}\left(2^{-k} \lambda\right)^{-n a} \\
& \quad \times \int_{I}\left|\left[b\left(x_{\omega}\right)-b\left(2^{-k} x+x_{\omega}\right)\right] f\left(2^{-k} x+x_{\omega}\right)\right|^{p} d x \\
& \leq C\left(2^{-k} \lambda\right)^{p(n-\alpha+\beta-n a / p)}\|b\|_{\dot{L}_{\beta}}^{p}\|f\|_{L^{p}(\omega)}^{p} .
\end{aligned}
$$

When $1<p<2$, using (1.4), we get

$$
\begin{aligned}
\int_{F(\omega)} \mid & \left.E(x, \omega)\right|^{p} d x \\
\leq & C \lambda^{p(n-\alpha+\beta)} 2^{-k p(n-\alpha+\beta)} 2^{-k n}\|b\|_{\dot{\Lambda}_{\beta}}^{p}\left(2^{-k} \lambda\right)^{-n a p / p^{\prime}} \int_{I}\left|f\left(2^{-k} x+x_{\omega}\right)\right|^{p} d x \\
& +C \lambda^{p(n-\alpha+\beta)} 2^{-k p(n-\alpha)} 2^{-k n}\left(2^{-k} \lambda\right)^{-n a p / p^{\prime}} \\
& \times \int_{I}\left|\left[b\left(x_{\omega}\right)-b\left(2^{-k} x+x_{\omega}\right)\right] f\left(2^{-k} x+x_{\omega}\right)\right|^{p} d x \\
\leq & C\left(2^{-k} \lambda\right)^{p\left(n-\alpha+\beta-n a / p^{\prime}\right)}\|b\|_{\dot{\Lambda}_{\beta}}^{p}\|f\|_{L^{p}(\omega)}^{p} .
\end{aligned}
$$

If $2 \leq p<p_{0}^{\prime}$, choose $\delta=n a / p-n+\alpha-\beta>0$. If $p_{0}<p<2$, choose $\delta=n a / p^{\prime}-n+\alpha-\beta>0$. Consequently, for $p_{0}<p<p_{0}^{\prime}$,

$$
\begin{aligned}
\sum_{k=0}^{k_{N}}\left\|E_{k}\right\|_{L^{p}(I)} & \leq C \sum_{k=0}^{k_{N}}\left(2^{-k} \lambda\right)^{-\delta}\|b\|_{\dot{\Lambda}_{\beta}}\|f\|_{L^{p}(I)} \\
& \leq \sum_{k=0}^{k_{N}}\left(2^{-k+k_{N}-1}\right)^{-\delta}\|b\|_{\dot{\Lambda}_{\beta}}\|f\|_{L^{p}(I)} \\
& \leq C\|b\|_{\dot{\Lambda}_{\beta}}\|f\|_{L^{p}(I)} .
\end{aligned}
$$

Therefore, using (2.13)-(2.15), we have

$$
\left\|S_{\lambda}^{b} f\right\|_{L^{p}(I)} \leq C \sum_{k=0}^{k_{N}}\left\|E_{k}\right\|_{L^{p}(I)}+C \sum_{k_{N}+1}^{\infty}\left\|E_{k}\right\|_{L^{p}(I)} \leq C\|b\|_{\dot{\Lambda}_{\beta}}\|f\|_{L^{p}(I)},
$$

where $p_{0}<p<p_{0}^{\prime}$ and $C$ is a constant independent of $\lambda$ and $f$. This implies (2.12). 
Finally, we will prove $\|[b, T] f\|_{L^{p_{0}\left(\mathbb{R}^{n}\right)}} \leq C\|b\|_{\dot{\Lambda}_{\beta}}\|f\|_{L^{p_{0}\left(\mathbb{R}^{n}\right)}}$ where $p_{0}=$ $n a /(n-\alpha+\beta)$. In fact,

$$
|[b, T] f(x)| \leq\|b\|_{\dot{\Lambda}_{\beta}} \int_{\mathbb{R}^{n}} \frac{|f(y)|}{|x-y|^{\alpha-\beta}} d y
$$

$=\|b\|_{\dot{\Lambda}_{\beta}} \int_{\mathbb{R}^{n}} e^{-i|x-y|^{a}} \frac{e^{i|x-y|^{a}}}{|x-y|^{\alpha-\beta}}|f(y)| d y$

$=\|b\|_{\dot{\Lambda}_{\beta}}\left[\int_{\mathbb{R}^{n}}\left(e^{-i|x-y|^{a}}-e^{i|x-y|^{a}}\right) \frac{e^{i|x-y|^{a}}}{|x-y|^{\alpha-\beta}}|f(y)| d y+\int_{\mathbb{R}^{n}} \frac{e^{2 i|x-y|^{a}}}{|x-y|^{\alpha-\beta}}|f(y)| d y\right]$

$=\|b\|_{\dot{\Lambda}_{\beta}}\left[-2 i \int_{\mathbb{R}^{n}}\left(\sin |x-y|^{a}\right) \frac{e^{i|x-y|^{a}}}{|x-y|^{\alpha-\beta}}|f(y)| d y+\int_{\mathbb{R}^{n}} \frac{e^{2 i|x-y|^{a}}}{|x-y|^{\alpha-\beta}}|f(y)| d y\right]$

$\leq 3\|b\|_{\dot{\Lambda}_{\beta}}\left|\int_{\mathbb{R}^{n}} \frac{e^{2 i|x-y|^{a}}}{|x-y|^{\alpha-\beta}}\right| f(y)|d y|$

$=3\|b\|_{\dot{\Lambda}_{\beta}} 2^{(\alpha-\beta-n) / a}\left|\int_{\mathbb{R}^{n}} \frac{e^{i\left|2^{1 / a} x-y\right|^{a}}}{\left|2^{1 / a} x-y\right|^{\alpha-\beta}}\right| f\left(2^{-1 / a} y\right)|d y|$.

Set $\tilde{f}(x)=f\left(2^{-1 / a} y\right)$ and

$$
T^{\alpha-\beta} f(x)=\int_{\mathbb{R}^{n}} \frac{e^{i|x-y|^{a}}}{|x-y|^{\alpha-\beta}} f(y) d y .
$$

Then by Theorem A (see [5]), $T^{\alpha-\beta}$ is bounded on $L^{p_{0}}\left(\mathbb{R}^{n}\right)$. It follows from (2.16) that

$$
|[b, T] f(x)| \leq C\|b\|_{\dot{\Lambda}_{\beta}}\left|T^{\alpha-\beta} \widetilde{f}\left(2^{1 / a} x\right)\right| .
$$

Hence,

$\|[b, T] f\|_{L^{p_{0}\left(\mathbb{R}^{n}\right)}} \leq C\|b\|_{\dot{\Lambda}_{\beta}}\left(\int_{\mathbb{R}^{n}}\left|T^{\alpha-\beta} \tilde{f}\left(2^{1 / a} x\right)\right|^{p_{0}} d x\right)^{1 / p_{0}} \leq C\|b\|_{\dot{\Lambda}_{\beta}}\|f\|_{L^{p_{0}\left(\mathbb{R}^{n}\right)}}$.

By duality, we complete the proof.

Acknowledgements. The authors would like to express their deep gratitude to the referees for some useful comments including the formulation of the main theorem of this paper.

\section{References}

[1] L. Carleson and P. Sjölin, Oscillatory integrals and a multiplier problem for the disc, Studia Math. 44 (1972), 287-299. 
[2] S. Janson, Mean oscillation and commutators of singular integral operators, Ark. Mat. 16 (1978), 263-270.

[3] M. Paluszyński, Characterization of the Besov spaces via the commutator operator of Coifman, Rochberg and Weiss, Indiana Univ. Math. J. 44 (1995), 1-17.

[4] G. Sampson, A. Naparstek and V. Drobot, $\left(L^{p}, L^{q}\right)$ mapping properties of convolution transforms, Studia Math. 55 (1976), 41-70.

[5] P. Sjölin, Convolution with oscillating kernels, Indiana Univ. Math. J. 30 (1981), $47-55$.

[6] E. M. Stein, Harmonic Analysis: Real-Variable Methods, Orthogonality, and Oscillatory Integrals, Princeton Univ. Press, Princeton, NJ, 1993.

Xia Xia

School of Mathematical Sciences

Beijing Normal University

Beijing, 100875, P.R. China

and

School of Mathematical Sciences

Tianjin Normal University

Tianjin, 300387, P.R. China

E-mail: xiaxiashuxue@sina.com
Shanzhen Lu School of Mathematical Sciences Beijing Normal University Beijing, 100875, P.R. China

E-mail: lusz@bnu.edu.cn

Received January 5, 2006

Revised version February 10, 2008 